\title{
Fluctuations of Chromodynamic Fields in Quark-Gluon Plasma *
}

\author{
Stanisław Mrówczyński \\ Institute of Physics, Jan Kochanowski University, ul. Świętokrzyska 15, 25-406 Kielce, Poland \\ and Soltan Institute for Nuclear Studies, ul. Hoża 69, 00-681 Warsaw, Poland
}

\begin{abstract}
Chromodynamic fluctuations in the collisionless quark-gluon plasma are found as a solution of the initial value linearized problem. The stable and unstable plasmas are discussed.

Key words: Quark-gluon plasma, kinetic theory, finite temperature field theory PACS: 12.38.Mh, 05.20.Dd, 11.10.Wx
\end{abstract}

In the quark-gluon plasma (QGP), which is on average locally colorless, chromodynamic fields, color charges and currents experience random fluctuations which appear to influence dynamics of the whole system. In the equilibrium plasmas there are characteristic stationary spectra of fluctuations which can be found by means of the fluctuationdissipation relations. Fluctuation spectra in nonequilibrium systems evolve in time, and their characteristics usually depend on an initial state of the system. Our aim is to discuss chromodynamic fluctuations in equilibrium and nonequilibrium QGP. We are particularly interested in QGP produced at the early stage of relativistic heavy-ion collisions. Such a plasma is unstable with respect to chromomagnetic modes due to anisotropic momentum distribution of quarks and gluons (partons), see the review [1. The instability growth is associated with generation of chromomagnetic fields which in turn influence various plasma properties. In particular, transport coefficients of such a plasma, which are controlled by the fluctuation spectrum of chromomagnetic fields, are then strongly modified [2].

Fluctuations can be theoretically studied by means of several methods reviewed in the classical monographs 34. We choose the method which is clearly exposed in the handbook [5]. The method - applicable to both equilibrium and nonequilibrium plasmas

ऋ Talk given at the Conference on Strong and Electroweak Matter 2008 (SEWM08), August 26-29, 2008, Amsterdam, The Netherlands 
- provides the spectrum of fluctuations as a solution of the initial value (linearized) problem. The initial plasma state is assumed to be on average charge neutral, stationary and homogeneous. When the state is stable, the initial fluctuations are explicitly shown to exponentially decay and in the long time limit one finds a stationary spectrum of the fluctuations. When the initial state is unstable, the memory of initial fluctuations is not lost, as the unstable modes, which are usually present in the initial fluctuation spectrum, exponentially grow. The fluctuations of chromodynamic fields in QGP have been discussed in detail in our study [6]. Here we briefly summarize the results.

The transport theory of weakly coupled quark-gluon plasma, which forms the basis of our analysis, is formulated in terms of particles and classical fields. The particles quarks, antiquarks and gluons - should be understood as sufficiently hard quasiparticle excitations of QCD quantum fields while the classical fields are highly populated soft gluonic modes. The transport equation of quarks reads

$$
\left(D^{0}+\mathbf{v} \cdot \mathbf{D}\right) Q(t, \mathbf{r}, \mathbf{p})-\frac{g}{2}\left\{\mathbf{E}(t, \mathbf{r})+\mathbf{v} \times \mathbf{B}(t, \mathbf{r}), \nabla_{p} Q(t, \mathbf{r}, \mathbf{p})\right\}=0,
$$

where $Q(t, \mathbf{r}, \mathbf{p})$ is the on-mss-shell quark distribution function which is $N_{c} \times N_{c}$ hermitean matrices belonging to the fundamental representation of the $\mathrm{SU}\left(N_{c}\right)$ group; the covariant derivative in the four-vector notation reads $D^{\mu} \equiv \partial^{\mu}-i g\left[A^{\mu}(x), \cdots\right]$ and $\mathbf{E}(t, \mathbf{r})$ and $\mathbf{B}(t, \mathbf{r})$ are the chromoelectric and chromomagnetic fields. The symbol $\{\ldots, \ldots\}$ denotes the anticommutator. Since the fluctuations of interest are assumed to be of the time scale, which is much shorter than that of inter-parton collisions, the collision terms are absent in Eq. (11). There are analogous transport equations for antiquark $(\bar{Q}(t, \mathbf{r}, \mathbf{p}))$ and gluon $(G(t, \mathbf{r}, \mathbf{p}))$ distribution functions.

The transport equations are supplemented by the Yang-Mills equations describing a self-consistent generation of the chromoelectric and chromomagnetic fields by the color four-current $j^{\mu}=(\rho, \mathbf{j})$

$$
j_{a}^{\mu}(t, \mathbf{r})=-g \int \frac{d^{3} p}{(2 \pi)^{3}} \frac{p^{\mu}}{E_{\mathbf{p}}} \operatorname{Tr}\left[\tau^{a}(Q(t, \mathbf{r}, \mathbf{p})-\bar{Q}(t, \mathbf{r}, \mathbf{p}))+T^{a} G(t, \mathbf{r}, \mathbf{p})\right],
$$

where $\tau^{a}, T^{a}$ with $a=1, \ldots, N_{c}^{2}-1$ are the $\mathrm{SU}\left(N_{c}\right)$ group generators in the fundamental and adjoint representations.

We consider small deviations from a stationary homogeneous state which is globally and locally colorless; there are no currents as well. The quark distribution function of this state is $Q_{n m}^{0}(\mathbf{p})=n(\mathbf{p}) \delta^{n m}$. Due to the absence of color charges and currents in the stationary and homogeneous state, the chromoelectric $\mathbf{E}(t, \mathbf{r})$ and chromomagnetic $\mathbf{B}(t, \mathbf{r})$ fields are expected to vanish while the potentials $A^{0}(t, \mathbf{r}), \mathbf{A}(t, \mathbf{r})$ are of pure gauge only. Since the plasma under considerations is assumed to be weakly coupled with the perturbative vacuum state, the potentials can be gauge away to vanish.

We write down the quark distribution function as $Q(t, \mathbf{r}, \mathbf{p})=Q^{0}(\mathbf{p})+\delta Q(t, \mathbf{r}, \mathbf{p})$, and we assume that $\left|Q^{0}\right| \gg|\delta Q|$ and $\left|\nabla_{p} Q^{0}\right| \gg\left|\nabla_{p} \delta Q\right|$ with the analogous formulas for antiquarks and gluons. We linearize the transport (10) and Yang-Mills equations in the deviations from the stationary homogeneous state. We assume that $\delta Q, \mathbf{E}, \mathbf{B}, A^{0}$ and $\mathbf{A}$ are all of the same order. The linearized transport equation is

$$
\left(\frac{\partial}{\partial t}+\mathbf{v} \cdot \nabla\right) \delta Q(t, \mathbf{r}, \mathbf{p})-g(\mathbf{E}(t, \mathbf{r})+\mathbf{v} \times \mathbf{B}(t, \mathbf{r})) \nabla_{p} n(\mathbf{p})=0 .
$$


The Yang-Mills equations get after the linearization the familiar form of Maxwell equations of multi-component electrodynamics.

The linearized transport and Maxwell equations are solved with the initial conditions $\delta Q(t=0, \mathbf{r}, \mathbf{p})=\delta Q_{0}(\mathbf{r}, \mathbf{p}), \mathbf{E}(t=0, \mathbf{r})=\mathbf{E}_{0}(\mathbf{r}), \mathbf{B}(t=0, \mathbf{r})=\mathbf{B}_{0}(\mathbf{r})$, by means of the one-sided Fourier transformation defined as

$$
f(\omega, \mathbf{k})=\int_{0}^{\infty} d t \int d^{3} r e^{i(\omega t-\mathbf{k} \cdot \mathbf{r})} f(t, \mathbf{r}) .
$$

The chromoelectric field, which solves the equations, is found as

$$
\begin{gathered}
{\left[-\mathbf{k}^{2} \delta^{i j}+k^{i} k^{j}+\omega^{2} \varepsilon^{i j}(\omega, \mathbf{k})\right] E_{a}^{j}(\omega, \mathbf{k})=-i \frac{g^{2}}{2} \int \frac{d^{3} p}{(2 \pi)^{3}} \frac{v^{i}\left(\mathbf{v} \times \mathbf{B}_{a 0}(\mathbf{k})\right)^{j} \nabla_{p}^{j} f(\mathbf{p})}{\omega-\mathbf{v} \cdot \mathbf{k}}} \\
-g \omega \int \frac{d^{3} p}{(2 \pi)^{3}} \frac{v^{i}}{\omega-\mathbf{k} \cdot \mathbf{v}} \delta N_{0}^{a}(\mathbf{k}, \mathbf{p})+i \omega E_{a 0}^{i}(\mathbf{k})-i\left(\mathbf{k} \times \mathbf{B}_{a 0}(\mathbf{k})\right)^{i}
\end{gathered}
$$

where $f(\mathbf{p}) \equiv n(\mathbf{p})+\bar{n}(\mathbf{p})+2 N_{c} n_{g}(\mathbf{p})$ and $\delta N_{0}^{a}(\mathbf{r}, \mathbf{p}) \equiv \operatorname{Tr}\left[\tau^{a}\left(\delta Q_{0}(\mathbf{r}, \mathbf{p})-\delta \bar{Q}_{0}(\mathbf{r}, \mathbf{p})\right)+\right.$ $\left.T^{a} \delta G_{0}(\mathbf{r}, \mathbf{p})\right] ; \varepsilon^{i j}(\omega, \mathbf{k})$ is the chromodielectric tensor of, in general, anisotropic plasma in the collisionless limit; $\varepsilon^{i j}(\omega, \mathbf{k})$ does not carry any color indices as it corresponds to a colorless state of the plasma.

When the plasma stationary state is isotropic, the dielectric tensor can be expressed through its longitudinal $\left(\varepsilon_{L}(\omega, \mathbf{k})\right)$ and transverse $\left(\varepsilon_{L}(\omega, \mathbf{k})\right)$ components and the matrix $\Sigma^{i j}(\omega, \mathbf{k}) \equiv-\mathbf{k}^{2} \delta^{i j}+k^{i} k^{j}+\omega^{2} \varepsilon^{i j}(\omega, \mathbf{k})$ from the left-hand-side of Eq. (2) can be inverted as

$$
\left(\Sigma^{-1}\right)^{i j}(\omega, \mathbf{k})=\frac{1}{\omega^{2} \varepsilon_{L}(\omega, \mathbf{k})} \frac{k^{i} k^{j}}{\mathbf{k}^{2}}+\frac{1}{\omega^{2} \varepsilon_{T}(\omega, \mathbf{k})-\mathbf{k}^{2}}\left(\delta^{i j}-\frac{k^{i} k^{j}}{\mathbf{k}^{2}}\right) .
$$

Then, Eq. (2) provides an explicit expression of the chromoelectric field. Using the Maxwell equations, the chromomagnetic field, color current and color density can be all expressed through the chromoelectric field.

The correlation functions $\left\langle E_{a}^{i}\left(t_{1}, \mathbf{r}_{1}\right) E_{b}^{j}\left(t_{2}, \mathbf{r}_{2}\right)\right\rangle,\left\langle B_{a}^{i}\left(t_{1}, \mathbf{r}_{1}\right) B_{b}^{j}\left(t_{2}, \mathbf{r}_{2}\right)\right\rangle$, where $\langle\cdots\rangle$ denotes averaging over statistical ensemble, are determined by the initial correlations such as $\left\langle\delta N_{0}^{a}\left(\mathbf{r}_{1}, \mathbf{p}_{1}\right) \delta N_{0}^{b}\left(\mathbf{r}_{2}, \mathbf{p}_{2}\right)\right\rangle,\left\langle E_{a 0}^{i}\left(\mathbf{r}_{1}\right) E_{b 0}^{j}\left(\mathbf{r}_{2}\right)\right\rangle,\left\langle\delta N_{0}^{a}\left(\mathbf{r}_{1}, \mathbf{p}_{1}\right) E_{b 0}^{j}\left(\mathbf{r}_{2}\right)\right\rangle$ which can be all expressed, using the Maxwell equations, through the correlation function of the distribution functions. The latter one is identified with the respective correlation function of the classical system of free quarks, antiquarks and gluons which on average is stationary and homogeneous. For quarks the correlation function is

$$
\begin{aligned}
\left\langle\delta Q^{m n}\left(t_{1}, \mathbf{r}_{1}, \mathbf{p}_{1}\right) \delta Q^{p r}\left(t_{2}, \mathbf{r}_{2}, \mathbf{p}_{2}\right)\right\rangle_{\text {free }} & =\delta^{m r} \delta^{n p}(2 \pi)^{3} \delta^{(3)}\left(\mathbf{p}_{1}-\mathbf{p}_{2}\right) \\
& \times \delta^{(3)}\left(\mathbf{r}_{2}-\mathbf{r}_{1}-\mathbf{v}_{1}\left(t_{2}-t_{1}\right)\right) n\left(\mathbf{p}_{1}\right) .
\end{aligned}
$$

In the case of equilibrium plasma where all collective modes are damped, we consider the times which are much longer than the decay time of collective excitations. Then, the correlation function of the chromoelectric fields equals

$$
\left\langle E_{a}^{i}\left(t_{1}, \mathbf{r}_{1}\right) E_{b}^{j}\left(t_{2}, \mathbf{r}_{2}\right)\right\rangle_{\infty}=\int \frac{d \omega}{2 \pi} \frac{d^{3} k}{(2 \pi)^{3}} e^{-i\left(\omega\left(t_{1}-t_{2}\right)-\mathbf{k} \cdot\left(\mathbf{r}_{1}-\mathbf{r}_{2}\right)\right)}\left\langle E_{a}^{i} E_{b}^{j}\right\rangle_{\omega \mathbf{k}},
$$


where the fluctuation spectrum is

$$
\left\langle E_{a}^{i} E_{b}^{j}\right\rangle_{\omega \mathbf{k}}=2 \delta^{a b} T \omega^{3}\left[\frac{k^{i} k^{j}}{\mathbf{k}^{2}} \frac{\Im \varepsilon_{L}(\omega, \mathbf{k})}{\left|\omega^{2} \varepsilon_{L}(\omega, \mathbf{k})\right|^{2}}+\left(\delta^{i j}-\frac{k^{i} k^{j}}{\mathbf{k}^{2}}\right) \frac{\Im \varepsilon_{T}(\omega, \mathbf{k})}{\left|\omega^{2} \varepsilon_{T}(\omega, \mathbf{k})-\mathbf{k}^{2}\right|^{2}}\right] .
$$

As seen, the fluctuation spectrum has strong peaks corresponding to the collective modes.

As an example of a nonequilibrium system, we discuss fluctuations of longitudinal chromoelectric fields in the two-stream system which is unstable with respect to longitudinal modes. Nonequlibrium calculations are much more difficult than the equilibrium ones. The first problem is to invert the matrix $\Sigma^{i j}(\omega, \mathbf{k})$. In the case of longitudinal electric field, which is discussed here, the matrix is replaced by the scalar function. The distribution function of the two-stream system is chosen to be

$$
f(\mathbf{p})=(2 \pi)^{3} n\left[\delta^{(3)}(\mathbf{p}-\mathbf{q})+\delta^{(3)}(\mathbf{p}+\mathbf{q})\right],
$$

where $n$ is the effective parton density in a single stream. There are four roots $\pm \omega_{ \pm}(\mathbf{k})$ of the dispersion equation $\varepsilon_{L}(\omega, \mathbf{k})=0$. The solution $\omega_{+}(\mathbf{k})$ represents the stable modes and $\omega_{-}(\mathbf{k})$ corresponds to the well-known two-stream electrostatic instability for $\mathbf{k}^{2}(\mathbf{k} \cdot \mathbf{u})^{2}<$ $2 \mu^{2}\left(\mathbf{k}^{2}-(\mathbf{k} \cdot \mathbf{u})^{2}\right)$ where $\mathbf{u} \equiv \mathbf{q} /|\mathbf{q}|$ is the stream velocity and $\mu^{2} \equiv g^{2} n /(2|\mathbf{q}|)$. Then, $\omega_{-}(\mathbf{k})=i \gamma_{\mathbf{k}}$ with $0 \leq \gamma_{\mathbf{k}} \in R$.

The correlation function of longitudinal chromoelectric fields generated by the unstable modes is found as

$$
\begin{aligned}
& \left\langle E_{a}^{i}\left(t_{1}, \mathbf{r}_{1}\right) E_{b}^{i}\left(t_{2}, \mathbf{r}_{2}\right)\right\rangle_{\text {unstable }}=\frac{g^{2}}{2} \delta^{a b} n \int \frac{d^{3} k}{(2 \pi)^{3}} \frac{e^{i \mathbf{k}\left(\mathbf{r}_{1}-\mathbf{r}_{2}\right)}}{\mathbf{k}^{2}} \frac{\left(\gamma_{\mathbf{k}}^{2}+(\mathbf{k} \cdot \mathbf{u})^{2}\right)^{2}}{\left(\omega_{+}^{2}-\omega_{-}^{2}\right)^{2} \gamma_{\mathbf{k}}^{2}} \\
& \times\left[\left(\gamma_{\mathbf{k}}^{2}+(\mathbf{k} \cdot \mathbf{u})^{2}\right) \cosh \left(\gamma_{\mathbf{k}}\left(t_{1}+t_{2}\right)\right)+\left(\gamma_{\mathbf{k}}^{2}-(\mathbf{k} \cdot \mathbf{u})^{2}\right) \cosh \left(\gamma_{\mathbf{k}}\left(t_{1}-t_{2}\right)\right)\right] .
\end{aligned}
$$

As seen, the correlation function of the unstable system is invariant with respect to space translations - it depends on the difference $\left(\mathbf{r}_{1}-\mathbf{r}_{2}\right)$ only. The plasma state, which is initially on average homogeneous, remains like this in course of the system's temporal evolution. The time dependence of the correlation function is very different from the space dependence. The electric fields exponentially grow and so does the correlation function both in $\left(t_{1}+t_{2}\right)$ and $\left(t_{1}-t_{2}\right)$. The fluctuation spectrum also evolves in time, as the growth rate of unstable modes is wave-vector dependent and after a sufficiently long time the fluctuation spectrum is dominated by the fastest growing modes.

\section{References}

[1] St. Mrówczyński, Acta Phys. Polon. B 37 (2006) 427.

[2] M. Asakawa, S. A. Bass and B. Muller, Prog. Theor. Phys. 116 (2007) 725.

[3] A.I. Akhiezer, I.A. Akhiezer, R.V. Polovin, A.G. Sitenko, and K.N. Stepanov, Plasma Electrodynamics (Pergamon, New York, 1975).

[4] A.G. Sitenko, Fluctuations and Non-Linear Wave Interactions in Plasmas, (Pergamon, Oxford, 1982).

[5] E.M. Lifshitz and L.P. Pitaevskii, Physical Kinetics (Pergamon Press, Oxford, 1981).

[6] St. Mrówczyński, Phys. Rev. D 77 (2008) 105022. 